See Article page 121.

\section{Commentary: Meta-analysis of postoperative radiotherapy in N2-positive lung cancer-Garbage in, garbage out?}

\author{
Kenneth P. Seastedt, MD, ${ }^{\mathrm{a}}$ and \\ Chuong D. Hoang, $\mathrm{MD}^{\mathrm{b}}$
}

Differences abound regarding the management of surgically resectable stage IIIA non-small cell lung cancer (NSCLC), as this clinicopathologic designation comprises a complex, highly heterogeneous group of patients. ${ }^{1}$ In Europe, primary operations for N2 disease may be more prevalent, whereas in North America there is a widely subscribed tendency to undergo lung surgery after induction chemoradiotherapy. ${ }^{2,3}$ In stage IIIA N2-positive patients with adjuvant chemotherapy after curative resection, the survival benefit of postoperative radiotherapy (PORT) has been a topic of ongoing debate.

In this issue of the Journal, Harling and colleagues ${ }^{4}$ provide an updated meta-analysis of NSCLC patients who have undergone more contemporary radiation therapy (RT) in the era of routine adjuvant chemotherapy. Ten studies reporting on treated patients from 1994 to 2011 were analyzed by random-effects modeling, and subgroup analysis was performed using meta-regression. The pooled dataset consisted of 18,077 patients, with $30.2 \%$ receiving PORT and $69.8 \%$ not receiving PORT. Three studies were prospective, and 3 were evaluations of the National Cancer Database at various historical time intervals. PORT was randomized in only in 2 studies ( $\mathrm{n}=172$ patients) that terminated early because of

\footnotetext{
From the a Department of Surgery, Uniformed Services University of the Health Sciences, F. Edward Hébert School of Medicine, Bethesda, Md; and ${ }^{\mathrm{b}}$ Thoracic Surgery Branch, Center for Cancer Research, National Cancer Institute, National Institutes of Health, Bethesda, Md.

Disclosures: The authors reported no conflicts of interest.

The Journal policy requires editors and reviewers to disclose conflicts of interest and to decline handling or reviewing manuscripts for which they may have a conflict of interest. The editors and reviewers of this article have no conflicts of interest.

Received for publication Dec 28, 2020; revisions received Dec 28, 2020; accepted for publication Jan 5, 2021; available ahead of print Feb 2, 2021.

Address for reprints: Chuong D. Hoang, MD, 10 Center Drive, Mail code 1201, Room 4-3940, Bethesda, MD 20892 (E-mail: chuong.hoang@nih.gov).

JTCVS Open 2021;5:133-4

2666-2736

Published by Elsevier Inc. on behalf of The American Association for Thoracic Surgery. This is an open access article under the CC BY-NC-ND license (http:// creativecommons.org/licenses/by-nc-nd/4.0/).

https://doi.org/10.1016/j.xjon.2021.01.003
}

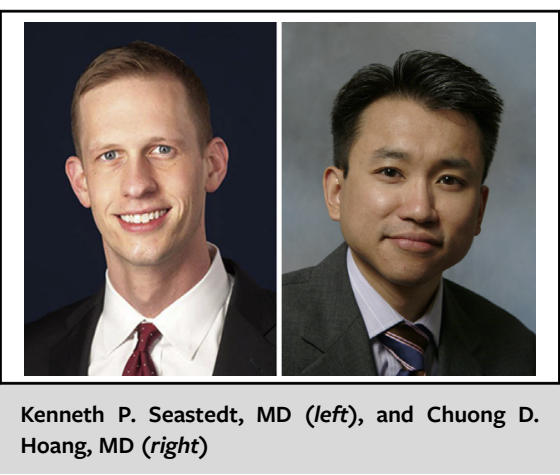

CENTRAL MESSAGE

Postoperative radiotherapy

(PORT) is shown to improve

survival in this updated meta-

analysis; however, multiple data

limitations soften the enthusiasm

for any true PORT benefit.

slow recruitment. Surgical resection details were available for only $27.6 \%$ of the patients, with $79.7 \%$ undergoing lobectomy, $11.3 \%$ undergoing sublobar resection, and $8.6 \%$ undergoing pneumonectomy. There were significantly more pneumonectomy patients in the no-PORT group $(P=.001)$ and more T3 patients in the PORT group $(P=.04)$. At 1,3 , and 5 years, the PORT group had significantly improved durations of overall survival (OS) and disease-free survival (DFS) compared with the no-PORT group. Meta-regression of multiple characteristics did not identify any independently significant associations with OS or DFS.

We congratulate the authors for their expert biostatistical assessment, but the many limitations inherent in the available datasets for this analysis should give everyone pause as to their overall conclusions. In addition to their acknowledgments, several broad limitations should be emphasized, including the lack of high-granularity data at the patient level stemming from retrospective collective analyses, the heterogeneity of both patient populations and therapeutic schemes stemming from changing clinical definitions over long time spans that persisted despite statistical adjustments, and, finally, the multiple unknown selection biases and unidentified sources of confounding from nonrandomized studies. It is prudent to keep in mind that nonrandomized studies tend to show larger treatment effects that in fact might not be true, and so pooling of these kinds of reports may not routinely arrive at high-confidence conclusions. Regarding specific caveats with this meta-analysis, most 
concerning is the lack of details on radiotherapy dose and planning techniques across the studies under review.

These limitations temper the strength of the authors' proPORT conclusions and recommendations. Early results from the international randomized Lung_ART trial (ClinicalTrials.gov identifier NCT00410683), directly studying modern mediastinal PORT, have not been formally published but were recently presented in an abstract. ${ }^{5}$ This prospective trial randomized 501 NSCLC patients (accrued from 2007 to 2018) after lung resection with pathologic N2 disease to PORT (54 Gy; 252 patients) or no PORT (249 patients), with most receiving chemotherapy (adjuvant, $77 \%$; neoadjuvant, $18 \%$ ). At a median follow-up of 4.8 years, preliminary findings showed no significant difference in 3-year DFS or OS between the study groups, with higher cardiopulmonary toxicity in the PORT group. Interestingly, the 3-year DFS and OS were higher than expected in both study arms, while mediastinal relapse was reduced by $50 \%$ in the PORT arm. The final results of this latest trial for PORT may provide succinct clinical guidance for this therapeutically challenging subgroup of NSCLC patients. It will be interesting to see whether future meta-analyses arrive at a different conclusion regarding the impact of PORT than that reached by Harling and colleagues.

\section{References}

1. Putora PM, Leskow P, McDonald F, Batchelor T, Evison M. International guidelines on stage III N2 non-small cell lung cancer: surgery or radiotherapy? ERJ Open Res. 2020;6:00159-2019.

2. Veeramachaneni NK, Feins RH, Stephenson BJ, Edwards LJ, Fernandez FG. Management of stage IIIA non-small cell lung cancer by thoracic surgeons in North America. Ann Thorac Surg. 2012;94:922-6; discussion 926-8.

3. Rocco G, Nason K, Brunelli A, Varela G, Waddell T, Jones DR. Management of stage IIIA (N2) non-small cell lung cancer: a transatlantic perspective. J Thorac Cardiovasc Surg. 2016;151:1235-8.

4. Harling L, Jayakumar S, Ashrafian H, Bille A, Toufektzian L, Smith D. Mediastinal radiotherapy after adjuvant chemotherapy for resected non-small cell lung cancer with N2 lymphadenopathy: a novel meta-analysis. J Thorac Cardiovasc Surg Open. 2021;5:121-30.

5. Le Pechoux C, Pourel N, Barlesi F, Faivre-Finn C, Lerouge D, Zalcman G, et al. LBA3_PR: an international randomized trial comparing postoperative conformal radiotherapy (PORT) to no PORT, in patients with completely resected non-small cell lung cancer (NSCLC) and mediastinal N2 involvement: primary end-point analysis of LungART (IFCT-0503, UK NCRI, SAKK) NCT00410683. Presidential Symposium II, 20 September 2020, ESMO Virtual Congress 2020. Available at: https://oncologypro.esmo.org/meeting-resources/esmo-virtual-congress-2020/ an-international-randomized-trial-comparing-post-operative-conformal-radiotherapyport-to-no-port-in-patients-with-completely-resected-non-smal. Accessed December $27,2020$. 